\title{
Analysis of the Impact of P-Ratio on BER, Q-Factor and OSNR of Radio over Fiber (RoF) System
}

\author{
Vishal Sharma ${ }^{1}$, Amarpal Singh ${ }^{2}$, Ajay K. Sharma ${ }^{3}$ \\ ${ }^{1}$ Shaheed Bhagat Singh College of Engineering and Technology, Ferozepur, India \\ ${ }^{2}$ Beant College of Engineering and Technology, Gurdaspur, India \\ ${ }^{3}$ Dr. B.R. Ambedkar NIT, Jalandhar, India \\ E-mail:er_vishusharma@yahoo.com \\ Received September 15, 2011; revised October 15, 2011; accepted November 6, 2011
}

\begin{abstract}
The impact of p-ratio i.e. ratio of carrier power to total received RF power at control office (CO) on Bit error rate (BER), Q- performance parameter and Optical signal noise ratio (OSNR) of RoF communication system is theoretically analyzed using angular full-linewidth at half maximum (FWHM) of Lorentzian shape for the $\mathrm{RF}$ oscillator in this paper. As reduction in Laser linewidth improves the performance of RoF system, the RF oscillator linewidth at FWHM also plays an important role in improving the performance of RoF system.
\end{abstract}

Keywords: RoF System, OSNR Parameter, BER, Q-Parameter, Fiber Dispersion, Matlab 7.0

\section{Introduction}

For realization of future high performance integrated networks, broadband distribution \& access networks and to meet the increasing demand of multimedia services with a guaranteed quality of service, RoF technology comes out as the most promising technology. RoF technology combines the capacity of optical networks with the flexibility and mobility of wireless networks. Reduction in complexity at the antenna site, reduction in installation cost of access networks, possibility of dynamically allocation of radio carriers to different antenna sites, transparency and scalability are the few advantages of RoF technology. These systems have several advantages including lower attenuation compared to the coaxial cable, higher bandwidth, immunity to the RF interference and durability [1-4]. Modulation technique is one of the most significant processes in RoF system where the RF electrical signal is applied to modulate the optical carrier. RoF modulation methods can be categorized into two main groups: direct modulation and external modulation. Direct modulation, a simple technique, directly modulates the amplitude of the laser beam but suffers from a laser-frequency chirp effect that degrades severely the performance of the system. However, this can be eliminated by using the external-modulation scheme [5] used to modulate the phase of the optical carrier. Furthermore, the conventional optical double sideband (ODSB) external modulation scheme degrades the received RF signal power due to fiber chromatic dispersion drastically. For frequency range beyond $5 \mathrm{GHz}$ external modulation is needed for higher speeds. No doubt such systems are capable of meeting the future requirements of High speed high data transfer services but some degradations effects the performance of such systems such as fiber dispersion and modulator's nonnearity. For overcoming this power degradation, an optical single sideband (OSSB) external modulation scheme is employed [5]. The effect of phase noises from a laser and an oscillator on OSSB-RoF system is analyzed and discussed [6]. Barry and Lee [7] and Salz [8] also analyzed the performance of coherent optical systems with laser phase noise by utilizing a wiener process as coherent detection provides better sensitivity than that of direct detection. A number of techniques have been invented to analyze and improve the performance of RoF system by mitigating these problems. Minimization of fiber dispersion using OSSB technique, reduction of laser linewidth [9] and using external modulators are the few important techniques. In this paper, we have analyzed theoretically the impact of p-ratio over performance parameters of RoF communication system using angular full-linewidth at half maximum (FWHM) of Lorentzian shape of the RF oscillator at different optical 
span and responsivity. In section II we present the theoretical analysis of OSNR for a radio over fiber (RoF) communication system. Section III presents results and discussion for system. Finally, section IV concludes the paper findings.

\section{Theoretical Analysis of OSNR in Rof System}

In a simple and compact architecture of RoF system as shown in Figure 1, the data is up-converted by a RF oscillator and optically modulated by a laser diode (LD) by means of a Mach Zehnder (MZM) external modulator in a control office (CO). The output signal of the MZM is transmitted via a standard single-mode fiber (SSMF) and detected by a PD to generate the photocurrent at a base station (BS). This photocurrent goes through a band-pass filter (BPF) and an amplifier to be launched into a wireless channel in the BS. The wireless channel makes signals vulnerable to amplitude and phase distortion. A user terminal (UT) amplifies and filters the received signal to detect the transmitted RF signal. Finally, the data are extracted through RF demodulation.

In this paper, an optical single sideband (OSSB) signal is generated by using a DEMZM external modulator together with a phase shifter to overcome the power deg radation due to fiber dispersion. This RF signal is optically modulated by the LD with a Dual electrode MZM external modulator. The optically modulated signal is transmitted to the PD and the photocurrent corresponding

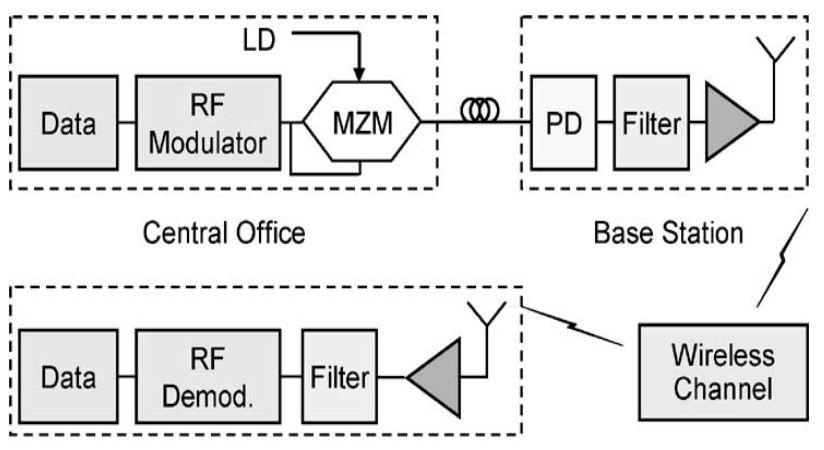

User Terminal

Figure1. Overall downlink architecture [6] of a RoF system. to the transmitted RF signal is extracted by the BPF. First, the optical signals from the laser and the RF oscillator are modeled as follows:

$$
\begin{aligned}
& x_{L D}(t)=A \cdot \exp j\left(\omega_{L D}+\phi_{L D}(t)\right) \\
& x_{R F}(t)=V_{R F} \cdot \cos j\left(\omega_{R F} t+\phi_{R F}(t)\right)
\end{aligned}
$$

where $A$ and $V_{\mathrm{RF}}$ define amplitudes from the LD and the $\mathrm{RF}$ oscillator, $\omega_{L D}$ and $\omega_{R F}$ define angular frequencies of the signals from the LD and the RF oscillator, and $\varphi_{L D}(t)$ and $\varphi_{R F}(t)$ are phase-noise processes. $\varphi_{L D}(t)$ is characterized by a Wiener process [7].

$$
\phi_{L D}(t)=\int_{0}^{t} \phi^{\prime}{ }_{L D}(\tau) \mathrm{d} \tau
$$

The time derivative $\varphi_{L D}^{\prime}(t)$ is not flat at low frequencies due to $1 / f$ noise. The white phase noise, however, is the principal cause for line broadening and is associated with quantum fluctuations [8]. Thus, $\varphi_{L D}^{\prime}(t)$ can be modeled as a zero-mean white Gaussian process with a power spectral density (PSD) function as

$$
S_{\phi^{\prime} L D}(\omega)=2 \pi \Delta v_{L D}
$$

where, $\Delta \mathrm{v}_{L D}$ defines a laser line-width. Unlike the phase-noise process from the laser, the phase-noise process $\varphi_{R F}(t)$ from the RF oscillator is difficult since the spectrum consists of various noises like flicker frequency, a white frequency noise, and a white phase noise. After optically modulating $x_{R F}(t)$ by $x_{L D}(t)$ with a DE-MZM, the output signal of the DE-MZM is represented [6] as (see Equation (4))

where $\tilde{x}_{R F}(t)$ denotes the phase-shift version of $x_{R F}(t)$, $\gamma=V_{d c} / V_{\pi}$ and $\alpha=V_{R F} / \sqrt{2} V_{\pi}$ define a normalized $\mathrm{dc}$ and ac value, $V_{\pi}$ is the switching voltage of the DEMZM, $L_{M Z M}$ is the insertion loss of the DEMZM, and $\theta$ is the phase shift by the phase shifter. Note that the input RF signals into the DEMZM are $x_{R F}(t) / \sqrt{2}$ and $\tilde{x}_{R F}(t) / \sqrt{2}$ rather than $x_{R F}(t)$ and $\tilde{x}_{R F}(t)$ because the $\mathrm{RF}$ signal is $3 \mathrm{~dB}$ attenuated by utilizing the power split ter. By controlling the phase shifter, the output signal can be the OSSB or the optical dual sideband (ODSB) signal. Among the two signals, only the OSSB signal will be dealt with in this paper since the ODSB signal suffers from fiber chromatic dispersion severely and requires double bandwidth

$$
\begin{aligned}
E(0, t)= & \frac{L_{M Z M} \cdot x_{L D}(t)}{\sqrt{2}}\left\{\exp j\left[\gamma \pi+\frac{\pi}{V_{\pi}} \cdot \frac{x_{R F}(t)}{\sqrt{2}}\right]+\exp j\left[\frac{\pi}{V_{\pi}} \cdot \frac{\tilde{x}_{R F}(t)}{\sqrt{2}}\right]\right\} \\
= & \frac{A \cdot L_{M Z M}}{\sqrt{2}}\left\{\exp j\left[\omega_{L D} t+\gamma \pi+\phi_{L D}(t)+\alpha \pi \cos \left(\omega_{R F} t+\phi_{R F}(t)\right)\right]\right. \\
& \left.+\exp j\left[\omega_{L D} t+\phi_{L D}(t)+\alpha \pi \cos \left(\omega_{R F} t+\phi_{R F}(t)+\theta\right)\right]\right\}
\end{aligned}
$$


than that of the OSSB signals. For generating the OSSB signal, $\theta$ and $\gamma$ are set to $\pi / 2$ and $1 / 2$, respectively. By using Equation (4) and the mentioned conditions, the OSSB signal at the DE MZM can be modeled as follows:

$$
\begin{aligned}
& E(0, t)=\frac{A \cdot L_{M Z M}}{\sqrt{2}}\left\{\exp j\left(\omega_{L D} t+\phi_{L D}(t)+\frac{\pi}{2}\right) \cdot \exp j\left(\alpha \pi \cos \left(\omega_{R F} t+\phi_{R F}(t)\right)\right)\right. \\
&+\left.\exp j\left(\omega_{L D} t+\phi_{L D}(t)\right) \cdot \exp j\left(\alpha \pi \cos \left(\omega_{R F} t+\phi_{R F}(t)+\frac{\pi}{2}\right)\right)\right\} \\
& E(0, t)=\frac{A \cdot L_{M Z M}}{\sqrt{2}}\left\{\exp j\left(\omega_{L D} t+\phi_{L D}(t)+\frac{\pi}{2}\right)\left[\cos \left(\alpha \pi \cos \left(\omega_{R F} t+\phi_{R F}(t)\right)\right)+j \sin \left(\alpha \pi \cos \left(\omega_{R F} t+\phi_{R F}(t)\right)\right)\right]\right. \\
&\left.+\exp j\left(\omega_{L D} t+\phi_{L D}(t)\right) \cdot\left[\cos \left(\alpha \pi \cos \left(\omega_{R F} t+\phi_{R F}(t)+\frac{\pi}{2}\right)\right)+j \sin \left(\alpha \pi \cos \left(\omega_{R F} t+\phi_{R F}(t)+\frac{\pi}{2}\right)\right)\right]\right\} \\
& \cos \left(\alpha \pi \cos \left(\omega_{R F} t+\phi_{R F}(t)\right)\right)=J_{0}(\alpha \pi)+2 \sum_{n=1}^{\infty} J_{2 n}(\alpha \pi) \cos 2 n\left(\omega_{R F} t+\phi_{R F}(t)\right) \\
& \sin \left(\alpha \pi \cos \left(\left(\omega_{R F} t+\phi_{R F}(t)\right)\right)=2 \sum_{n=0}^{\infty}(-1)^{n} J_{2 n+1}(\alpha \pi) \cos (2 n+1)\left[\omega_{R F} t+\phi_{R F}(t)\right]\right. \\
& \cos \left(\alpha \pi \sin \left(\omega_{R F} t+\phi_{R F}(t)\right)\right)=J_{0}(\alpha \pi)+2 \sum_{n=1}^{\infty} J_{2 n}(\alpha \pi) \cos 2 n\left(\omega_{R F} t+\phi_{R F}(t)\right) \\
& \sin \left(\alpha \pi \sin \left(\omega_{R F} t+\phi_{R F}(t)\right)\right)=2 \sum_{n=0}^{\infty} J_{2 n+1}(\alpha \pi) \cos (2 n+1)\left[\omega_{R F} t+\phi_{R F}(t)\right]
\end{aligned}
$$

Now by using the Jacobi's expansion for cos- and sin-terms and after simplification, Equation (5) can be written as

$$
E_{S}(0, t) \cong A \cdot L_{M Z M} \cdot\left\{J_{0}(\alpha \pi) \cdot \exp j\left(\omega_{L D} t+\phi_{L D}(t)+\frac{\pi}{4}\right)-\sqrt{2} J_{1}(\alpha \pi) \times \exp j\left(\omega_{L D} t+\omega_{R F} t+\phi_{L D}(t)+\phi_{R F}(t)\right)\right\}
$$

By neglecting the high-order components of the Bessel function since the value of $\alpha \pi$ in the Bessel function is very small due to the fact that $V_{\pi} \gg V_{R F}$ in general. The output signal at the DEMZM is transmitted via the SSMF

$$
\begin{aligned}
E_{S}(L, t)= & A \cdot L_{M Z M} \cdot L_{a d d} \cdot 10^{-\frac{\alpha_{\text {fiber }} \cdot L_{\text {Fiber }}}{20}} \cdot J_{0}(\alpha \pi) \\
& \times\left\{\exp j\left(\omega_{L D} t+\phi_{L D}\left(t-\tau_{0}\right)-\phi_{1}+\frac{\pi}{4}\right)-\frac{\sqrt{2} J_{1}(\alpha \pi)}{J_{0}(\alpha \pi)} \exp j\left(\omega_{L D} t+\omega_{R F} t+\phi_{L D}\left(t-\tau_{+}\right)+\phi_{R F}\left(t-\tau_{+}\right)-\phi_{2}\right)\right\}
\end{aligned}
$$

where $L_{\text {add }}$ denotes an additional loss in the optical link, $\alpha_{\text {fiber }}$ is the SSMF loss, $L_{\text {Fiber }}$ is the transmission distance of the SSMF, $\tau_{0}$ and $\tau_{+}$define group delays for a center angular frequency of $\omega_{L D}$ and an upper sideband frequency of $\omega_{L D}+\omega_{R F}, \phi_{1}$ and $\phi_{2}$ are phase-shift parameters for specific frequencies due to the fiber experiencing different group delays, due to the fiber chromatic dispersion, at a different wavelength. After the transmission of $L_{\text {Fiber }} \mathrm{km}$, the signal at the end of the SSMF becomes

$$
i(t)=\Re\left|E_{S}(L, t)\right|^{2}=\Re \cdot A_{1}^{2}\left[B+2 \alpha_{1} \cos \left(\omega_{R F}+\phi_{L D}\left(t-\tau_{+}\right)-\phi_{L D}\left(t-\tau_{0}\right)+\phi_{R F}\left(t-\tau_{+}\right)-\phi_{2}+\phi_{1}\right)\right]
$$

where

$$
\begin{aligned}
& A_{1}=A \cdot L_{M Z M} L_{\text {add }} 10^{-\frac{\alpha_{\text {Fiber }} . L_{\text {Fiber }}}{20}} J_{0}(\alpha \pi), \alpha_{1}=\frac{\sqrt{2} J_{1}(\alpha \pi)}{J_{0}(\alpha \pi)}, \\
& B=1+\alpha_{1}^{2}
\end{aligned}
$$

chromatic dispersion. For performance evaluation of RoF system, we determine and analyze OSNR performance parameter by employing the ratio between the signal power and the noise power by using an autocorrelation and a PSD- function. By using a square-law model, the photocurrent $i(t)$ can be obtained from (7) as follows:

where, $\Re$ defines the responsivity of the PD and $\mid f^{2}$ is the square-law detection. From Equation (8), the autocorrelation function $R_{I}(\tau)$ is obtained as

$$
R_{I}(\tau)=\langle i(t) \cdot i(t+\tau)\rangle
$$




$$
\frac{R_{I}(\tau)}{\mathfrak{R}^{2} \cdot A_{1}^{4}}=B^{2}+\left\{\begin{array}{l}
2 \alpha_{1}^{2} \cos \left(\omega_{R F} \tau\right) e^{-2 \gamma_{e}|\tau|},|\tau| \leq \tau_{1} \\
2 \alpha_{1}^{2} \cos \left(\omega_{R F} \tau\right) e^{-2 \gamma_{L D} \tau_{1}-2 \gamma_{R F}|\tau|},\left|\tau>\tau_{1}\right|
\end{array}\right.
$$

where, $\Delta v_{L D}$ and $\Delta v_{R F}$ are the laser- and RF oscillator- line-width respectively and $2 \gamma_{L D}=\left(2 \pi \Delta v_{L D}\right)$ and $2 \gamma_{R F}=\left(2 \pi \Delta v_{R F}\right)$ define the angular full line-width at half maximum (FWHM) of the Lorentzian shape for the laser and the RF oscillator, respectively. The $2 \gamma_{t}$, termed as total line-width, is not given as $2 \pi \Delta v_{L D}+2 \pi \Delta v_{R F}$ but

$$
\begin{aligned}
S_{I}(f)= & F\left\langle R_{I}(\tau)\right\rangle \frac{S_{I}(f)}{\mathfrak{R}^{2} \cdot A_{1}^{4}} \\
= & B^{2} \delta(f)+\frac{2 \gamma_{R F} \alpha_{1}^{2} \cdot e^{-2 \gamma_{e} \tau_{1}} \cos \left[2 \pi\left(f-f_{R F}\right) \tau_{1}\right]}{\gamma_{R F}^{2}+\left[2 \pi\left(f-f_{R F}\right)\right]^{2}}+\frac{4 \alpha_{1}^{2} \cdot e^{-2 \gamma_{t} \tau_{1}}}{\left(2 \gamma_{t}\right)^{2}+\left[2 \pi\left(f-f_{R F}\right)\right]^{2}} \\
& \cdot\left\{\gamma_{t} \cdot e^{2 \gamma_{t} \tau_{1}}-\gamma_{t} \cos \left[2 \pi\left(f-f_{R F}\right) \tau_{1}\right]-\frac{4 \pi \cdot \gamma_{L D}\left(\gamma_{L D}+\gamma_{R F}\right)\left(f-f_{R F}\right)}{\gamma_{R F}^{2}+\left[2 \pi\left(f-f_{R F}\right)\right]^{2}} \cdot \sin \left[2 \pi\left(f-f_{R F}\right) \tau_{1}\right]\right\}+G\left(f+f_{R F}\right)
\end{aligned}
$$

The first term of Equation (12) represents a dc component, the second and third is the broadening effects due to the fiber chromatic dispersion and the line-widths of as $2 \pi \Delta v_{L D}+\pi \Delta v_{R F}$. The term $\tau_{1}$ is the differential delay due to the fiber chromatic dispersion and is dependent on the optical wavelength $\lambda$, carrier frequency $f_{R F}$, fiber dispersion parameter, $D$ and optical length, $L_{F i b e r}$ and mathematically, given [10] as

$$
\tau_{1}=D \cdot L_{F i b e r} \cdot \lambda^{2} \cdot \frac{f_{R F}}{c}
$$

where, $\mathrm{c}$ is the light velocity. The PSD function of the The PSD function $S_{I}(f)$ can be written as photocurrent is given by the Fourier transform of (8).

$$
\begin{aligned}
P_{I}= & 2 \int_{f_{R F}-\frac{B_{R F}}{2}}^{f_{R F}} S_{I}(f) \mathrm{d} f \\
P_{I}= & 2 \times \Re^{2} \cdot A_{1}^{4}\left\{\int_{f_{R F}-\frac{B_{R F}}{2}}^{2} B^{2} \delta(f) \mathrm{d} f+\int_{f_{R F} \frac{B_{R F}}{2}}^{f_{R F}} \frac{B_{R F}}{2} \frac{2 \gamma_{R F} \alpha_{1}^{2} \cdot e^{-2 \gamma_{t} \tau_{1}} \cdot \cos \left(2 \pi\left(f-f_{R F}\right) \tau_{1}\right.}{\gamma_{R F}^{2}+\left(2 \pi\left(f-f_{R F}\right)\right)^{2}} \cdot \mathrm{d} f+\int_{f_{R F}-\frac{B_{R F}}{2}}^{f_{R F}+\frac{B_{R F}}{2}} \frac{4 \alpha_{1}^{2} \cdot e^{-2 \gamma_{t} \tau_{1}}}{\left(2 \gamma_{t}\right)^{2}+\left(2 \pi\left(f-f_{R F}\right)\right)^{2}}\right. \\
& \left.\cdot\left[\gamma_{t} \cdot e^{2 \gamma_{t} \tau_{1}}-\gamma_{t} \cos \left(2 \pi\left(f-f_{R F}\right) \tau_{1}\right)-\frac{4 \pi \cdot \gamma_{L D}\left(\gamma_{L D}+\gamma_{R F}\right)\left(f-f_{R F}\right)}{\gamma_{R F}^{2}+\left(2 \pi\left(f-f_{R F}\right)\right)^{2}} \cdot \sin \left(2 \pi\left(f-f_{R F}\right) \tau_{1}\right)\right]\right\} \\
\cong & \frac{4 \Re^{2} A_{1}^{4} \alpha_{1}^{2}}{\pi}\left\{\left(1-e^{-2 \gamma_{t} \tau_{1}}\right) \tan ^{-1}\left(\frac{\pi \cdot B_{R F}}{2 \gamma_{t}}\right)+e^{-2 \gamma_{t} \tau_{1}} \cdot \tan ^{-1}\left(\frac{\pi \cdot B_{R F}}{\gamma_{R F}}\right)\right\} \\
\cong & \frac{4 \Re^{2} A_{1}^{4} \alpha_{1}^{2}}{\pi} e^{-2 \gamma_{t} \tau_{1}} \cdot \tan ^{-1}\left(\frac{\pi \cdot B_{R F}}{\gamma_{R F}}\right) \quad P_{t}=R_{I}(0)-\mathfrak{R}^{2} \cdot A_{1}^{4} \cdot B^{2}=2 \mathfrak{R}^{2} A_{1}^{4} \alpha_{1}^{2}
\end{aligned}
$$
$\gamma_{t} \ll \gamma_{R F}$, is reasonable because the laser line- width is much greater than the RF-oscillator line- width and the fiber length is usually less than a few tens of kilometers in RoF systems. Note that the coefficient 2 of Equation (13) is due to the real and imaginary spectrum. The cos and sin terms in Equation (12) are approximately equal to 1 and 0 in the integrand when $\pi B_{R F} \tau_{1} \ll 1$ is satisfied.The received RF signal power is a function of differential delay $\tau_{1}$, laser- and RF oscillatorline-width and bandwidth of electrical filter $B_{R F}$.

For evaluating the total RF power excluding dc power, we utilize Equation (10) as follows: the laser and the RF oscillator. By using Equation (12), the received RF carrier power $P_{I}$ is approximately represented as follows [6]
By using (14), we define the ratio $p$ between the total carrier power and the required power as follows [6]:

$$
\begin{aligned}
p & =\frac{P_{I}}{P_{t}} \\
& =\frac{\frac{4 \Re^{2} A_{1}^{4} \alpha_{1}^{2}}{\pi} e^{-2 \gamma_{t} \tau_{1}} \cdot \tan ^{-1}\left(\frac{\pi \cdot B_{R F}}{\gamma_{R F}}\right)}{2 \mathfrak{R}^{2} A_{1}^{4} \alpha_{1}^{2}} \\
& =\frac{2}{\pi} e^{-2 \gamma_{t} \tau_{1}} \cdot \tan ^{-1}\left(\frac{\pi \cdot B_{R F}}{\gamma_{R F}}\right)
\end{aligned}
$$

for $2 \gamma_{t} \tau_{1} \ll 1$ and $\gamma_{t} \ll \gamma_{R F}$ 
From (14), the required bandwidth for the $p$ - ratio is obtained as

$$
B_{R F}=\frac{\gamma_{R F}}{\pi} \cdot \tan \left(\frac{\pi}{2} e^{2 \gamma_{t} \tau_{1}} p\right)
$$

The required bandwidth increases as we need more received signal power. Note that the required bandwidth for a specific received carrier power is dominantly dependent on the phase noise from the RF oscillator rather than that from the laser for $2 \gamma_{t} \tau_{1} \ll 1$ and $\gamma_{t} \ll \gamma_{R F}$. By using Equations (14) and (16), OSNR is calculated as

\section{OSNR}

$$
\begin{aligned}
& =\frac{\text { Signal }_{\text {power }}}{\text { Noise }_{\text {power }}}=\frac{P_{t}}{\text { Noise }_{\text {power }}}=\frac{2 \mathfrak{R}^{2} A_{1}^{4} B^{2}}{2 B_{R F} \cdot \frac{N_{0}}{2}} \\
& =\frac{\frac{1}{2} \cdot \frac{\left(\mathfrak{R} \cdot V_{r f} \cdot \pi\right)^{2}}{\left(V_{\pi}\right)^{2}}\left[A \cdot L_{M Z M} \cdot L_{\text {add }} \cdot 10 \frac{\alpha_{\text {fiber }} \cdot L_{\text {Fiber }}}{20}\right]^{4}}{20 N_{0} \cdot \tan \left(\frac{\pi \cdot p \cdot e^{2 \gamma_{t} \tau_{1}}}{2}\right)}
\end{aligned}
$$

The value of Bit error rate (BER) by using the direct relation among BER, Q-parameter and OSNR [10] can be predicted as

$$
\begin{aligned}
\mathrm{BER} & =\text { Error probability of } \mathrm{Q} \\
& =\frac{1}{\sqrt{\pi}} \int_{\frac{Q}{\sqrt{2}}}^{\infty} e^{-x^{2}} \cdot \mathrm{d} x=\frac{1}{2}\left[1-\operatorname{erf}\left(\frac{Q}{\sqrt{2}}\right)\right] \\
& =\frac{1}{\sqrt{2 \pi}} \cdot \frac{e^{-\frac{Q^{2}}{2}}}{Q}
\end{aligned}
$$

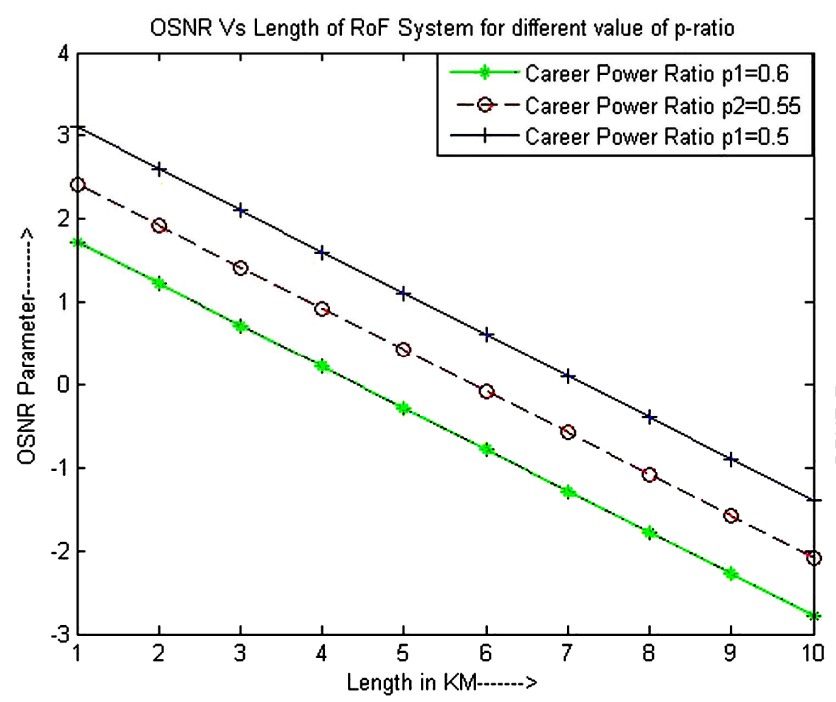

(a) where,

$$
\mathrm{Q}=\frac{2 \sqrt{2} \cdot \mathrm{OSNR}}{1+\sqrt{1+4 \mathrm{OSNR}}}
$$

\section{Results and Discussion}

The impact of $\mathrm{p}$ ratio using angular full-linewidth at half maximum (FWHM) of Lorentzian shape for the RF oscillator can be easily observed graphically of the RoF system at different optical span by varying responsivity of photo detector. The OSNR decreases as we increases the optical span but reduces as we decreases the $\mathrm{p}$ ratio from $p=0.6$ to $p=0.5$ as shown in Figures 2-3. Also it is observed that OSNR increases with increasing the responsivity (R-parameter) of the photodetector along with by using the angular full-linewidth at half maximum (FWHM) of Lorentzian shape the RF oscillator. The OSNR increases about $3 \mathrm{dBm}$ by using the RF oscillator linewidth at FWHM as shown in Figures 2(a) and 3(a).

The performance parameter $\mathrm{Q}$ also increases by decreasing the p-ratio of the RoF system and by increasing the R-parameter. The Q-parameter is also improving by using the RF oscillator linewidth at FWHM as shown in Figures 4-5. The BER of RoF system is also improving in the same manner depending upon the parameter such as R-parameter, p-ratio and gamma RF as shown in Figures 6-9. The results obtained and shown graphically if Figures 2-9 reveals that the performance of the RoF system improves if the RF oscillator is defined at angular full-linewidth at half maximum (FWHM) with increasing the R-parameter.

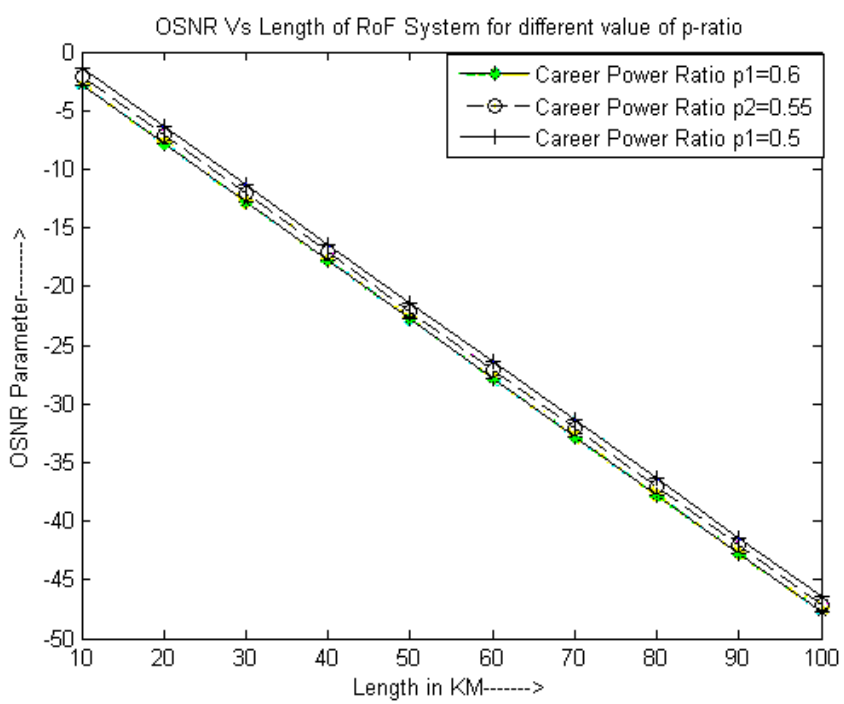

(b) 


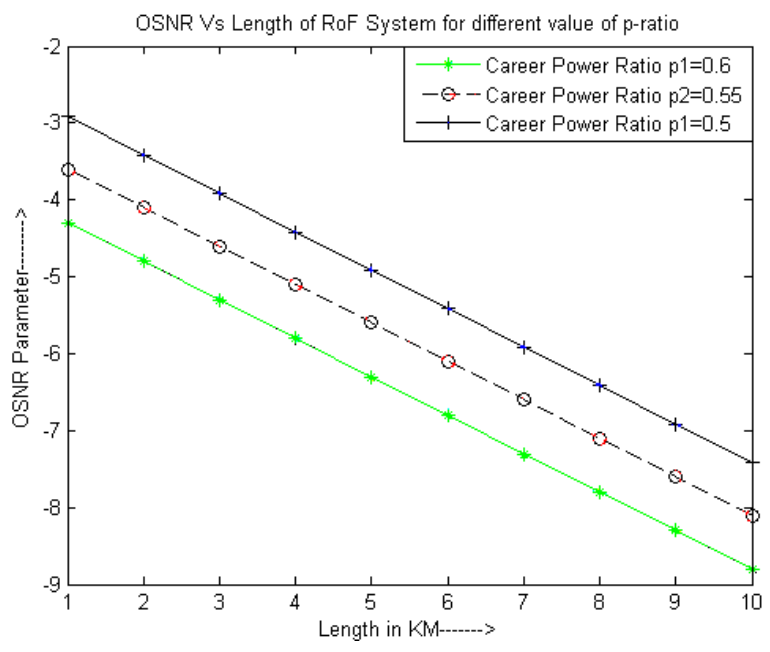

(c)

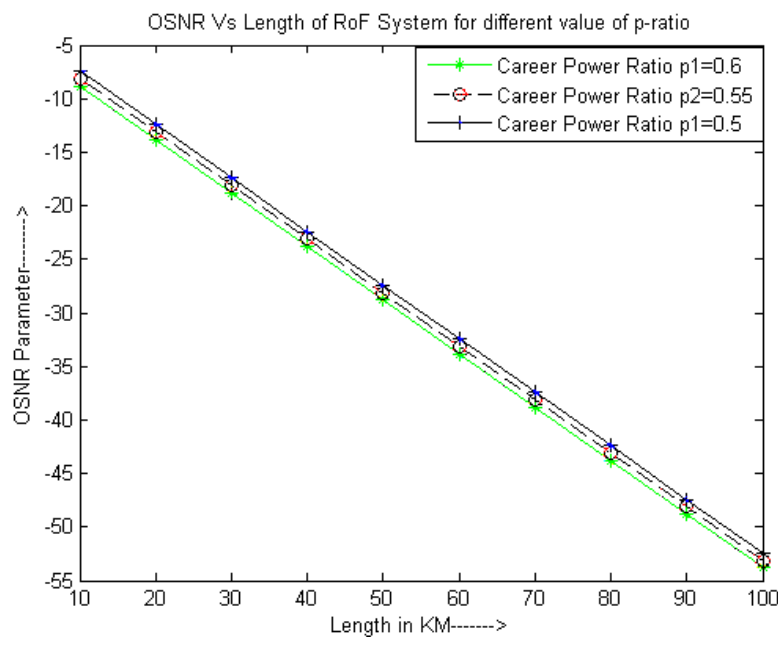

(d)

Figure 2. OSNR vs optical length for $(a, b) R=1$ and $(c, d) R=0.5$ with $\gamma_{R F}=\pi$.

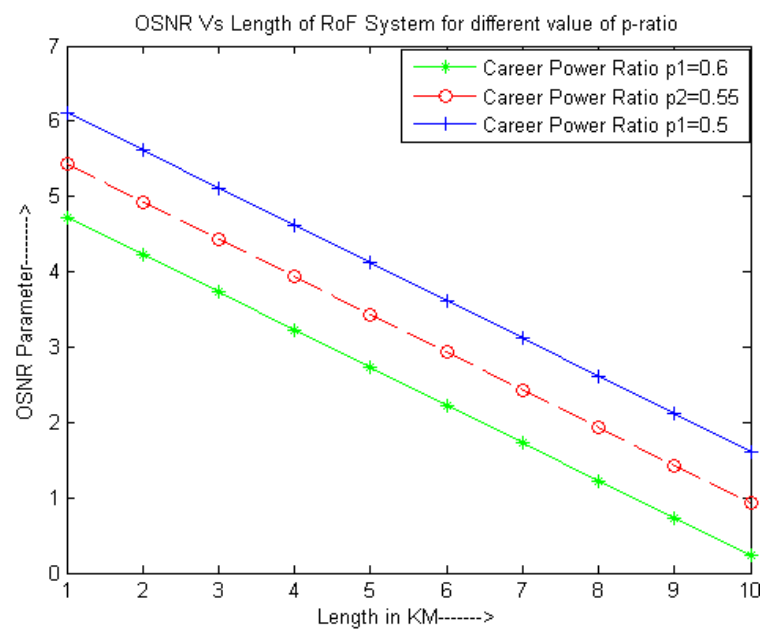

(a)

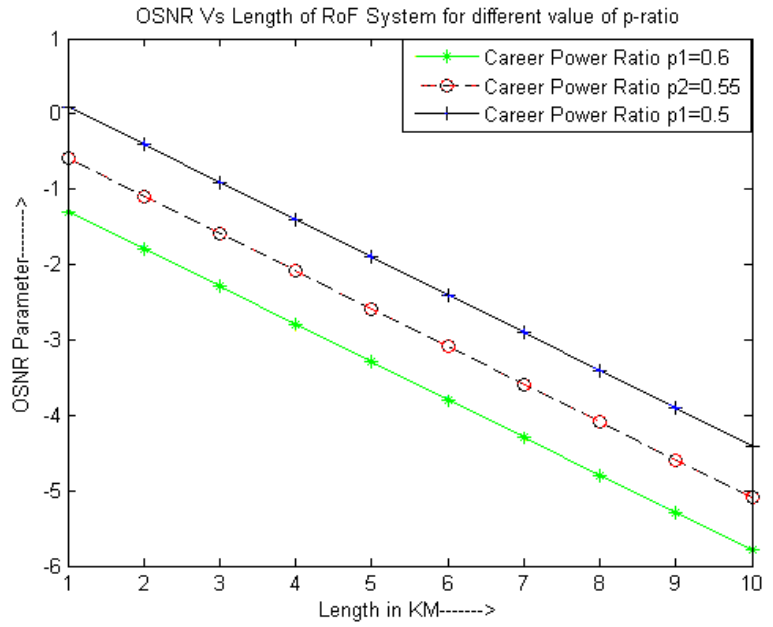

(c)

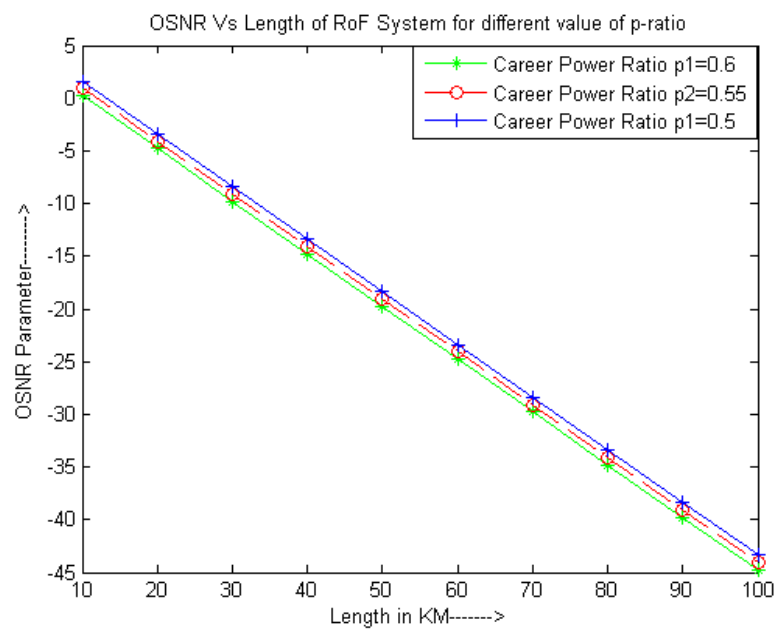

(b)

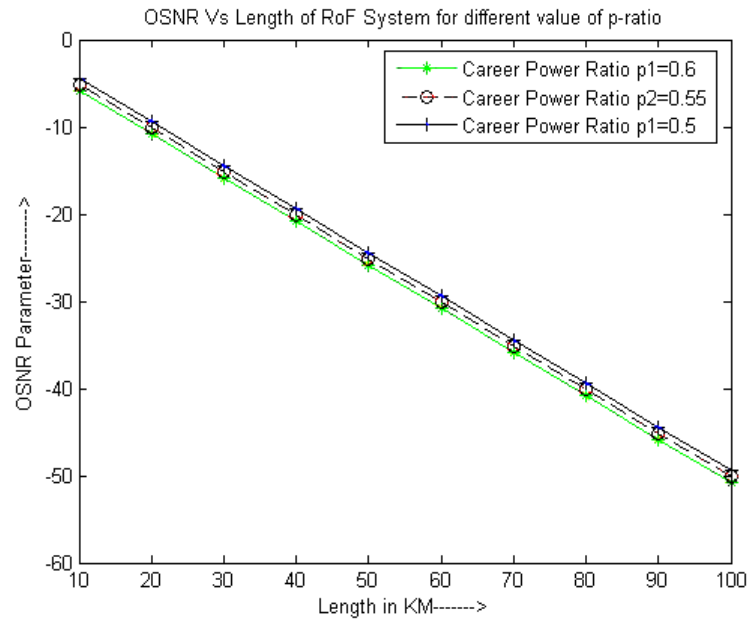

(d)

Figure 3. OSNR vs optical length for (a,b) $R=1$ and (c,d) $R=0.5$ with $\gamma_{R F}=\pi / 2$. 


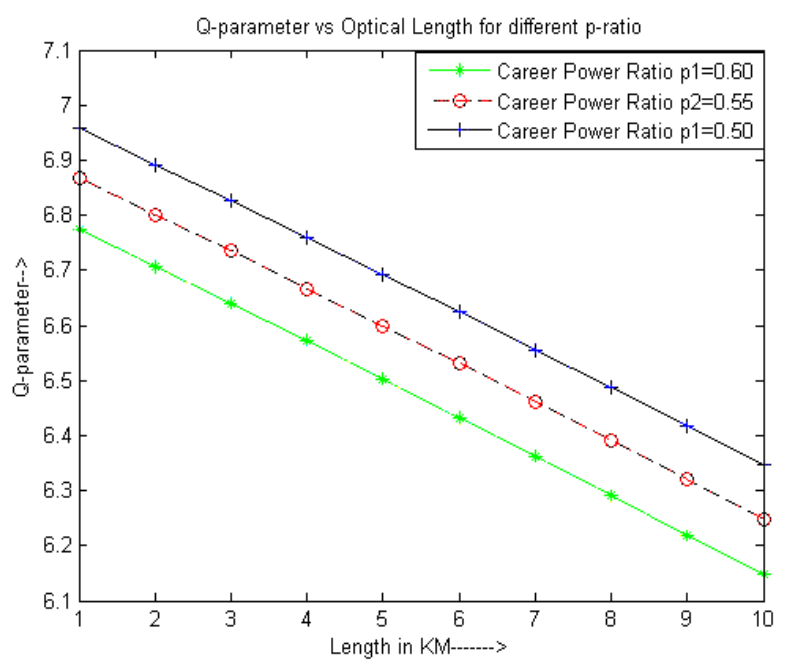

(a)

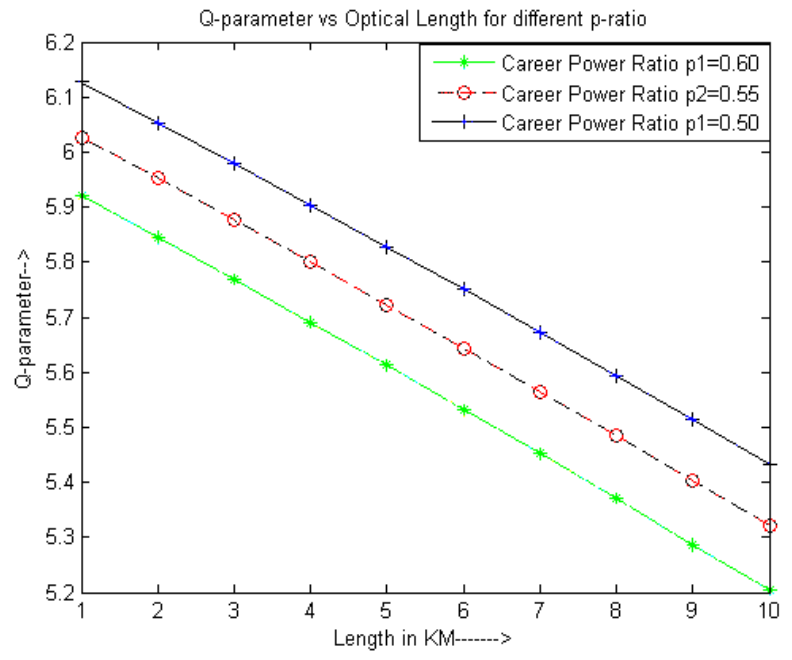

(c)

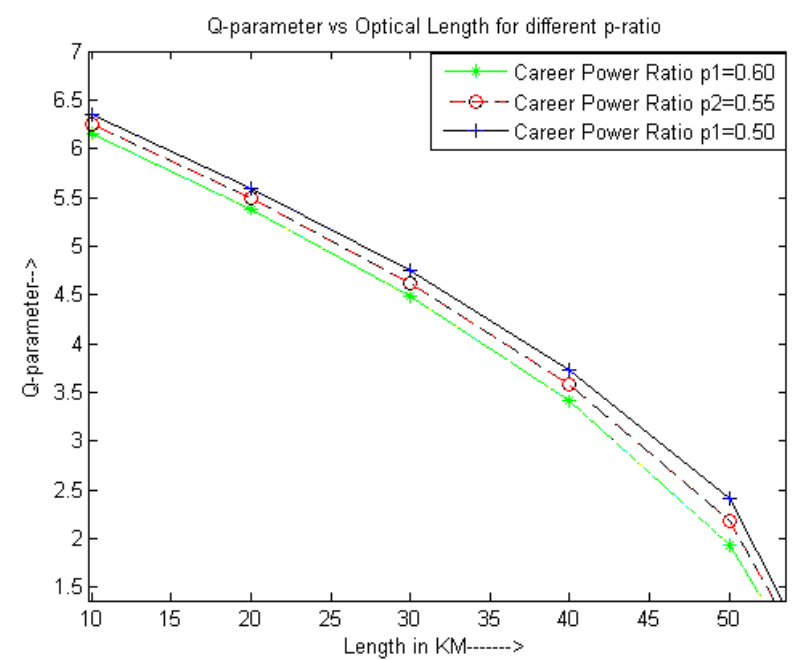

(b)

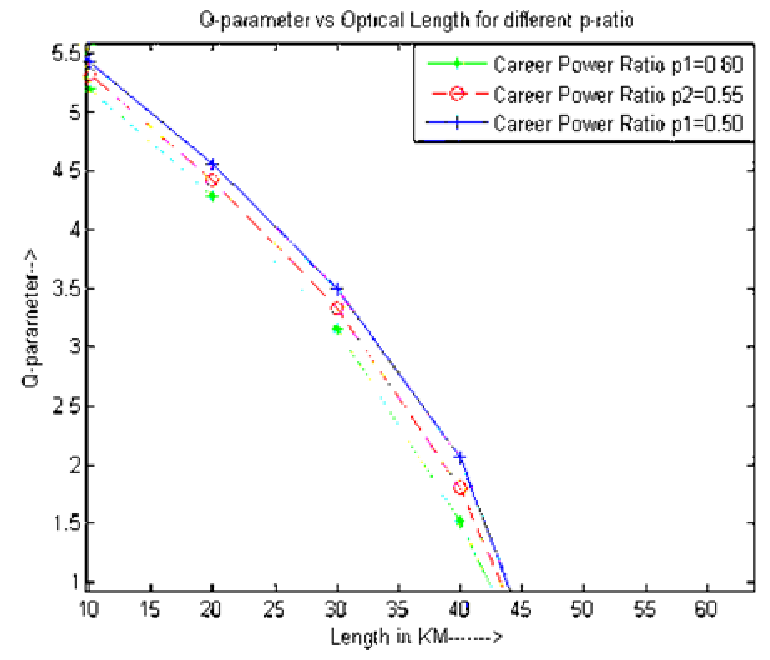

(d)

Figure 4. Q-parameter vs optical length for $(a, b) R=1$ and $(c, d) R=0.5$ with $\gamma_{R F}=\pi$.

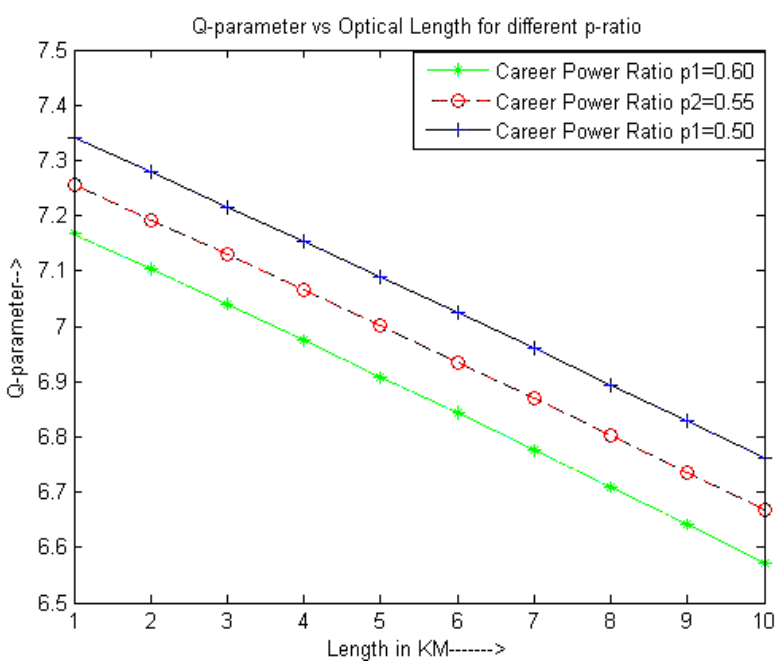

(a)

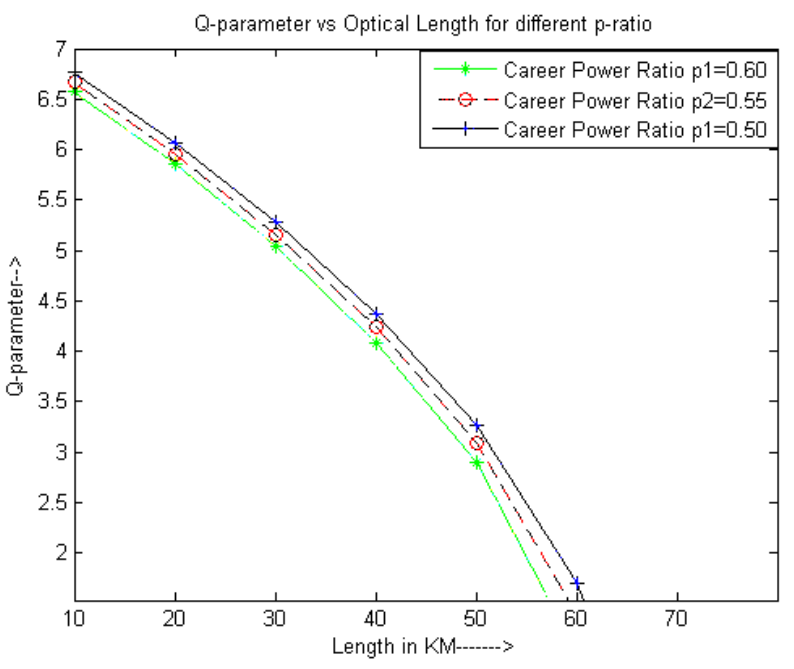

(b) 


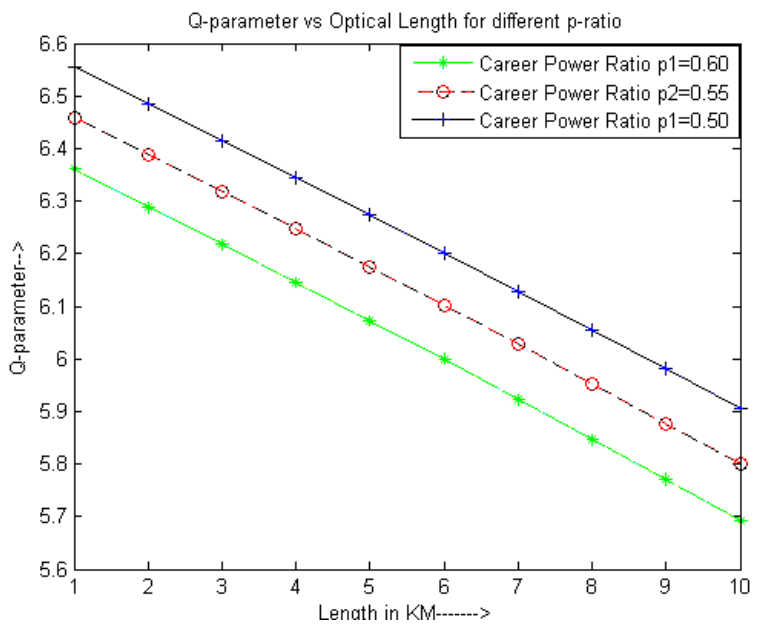

(c)

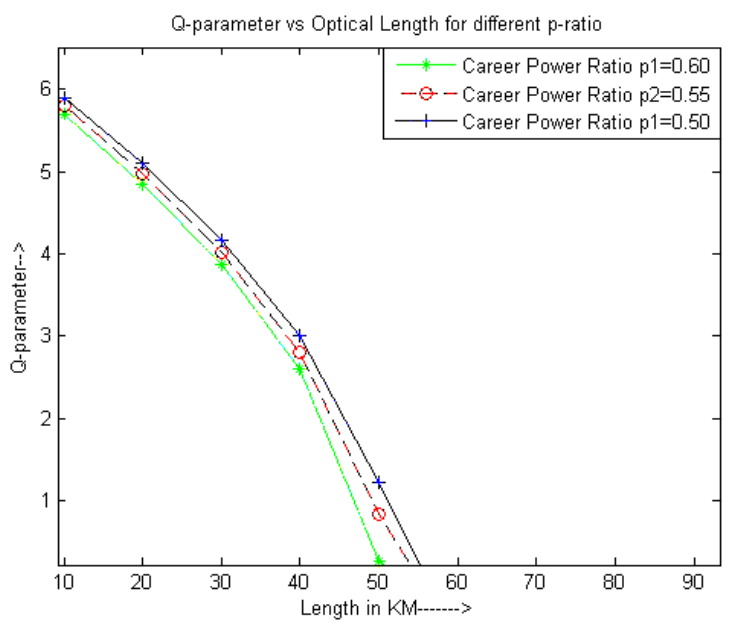

(d)

Figure 5. Q-parameter vs optical length for $(a, b) R=1$ and $(c, d) R=0.5$ with $\gamma_{R F}=\pi / 2$.

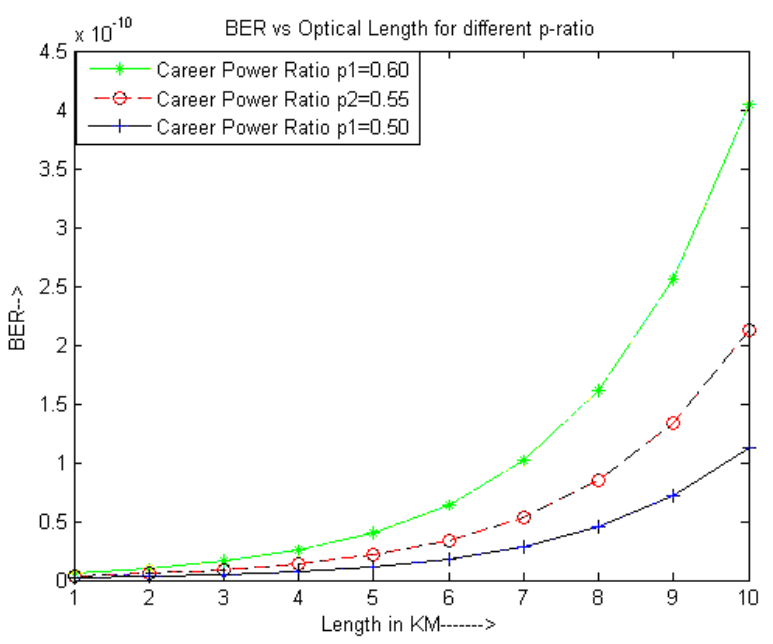

(a)

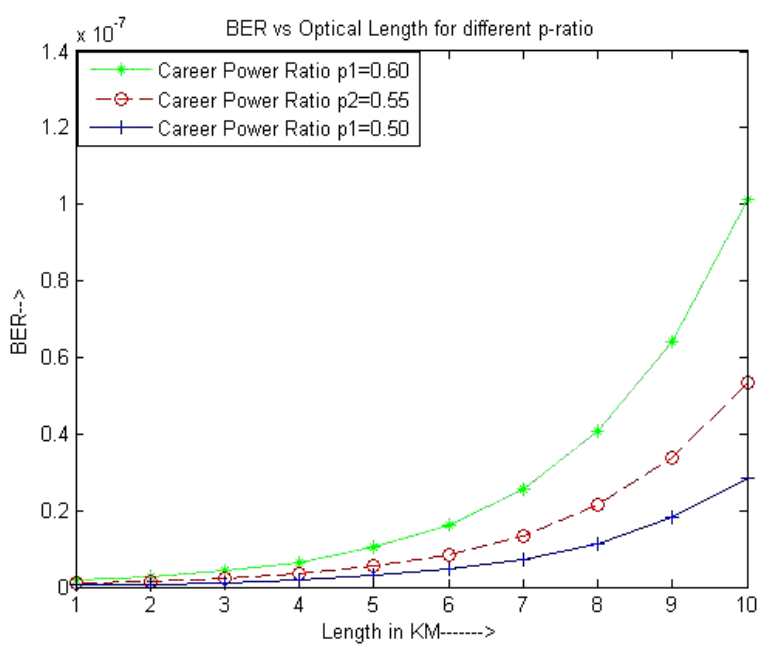

(c)

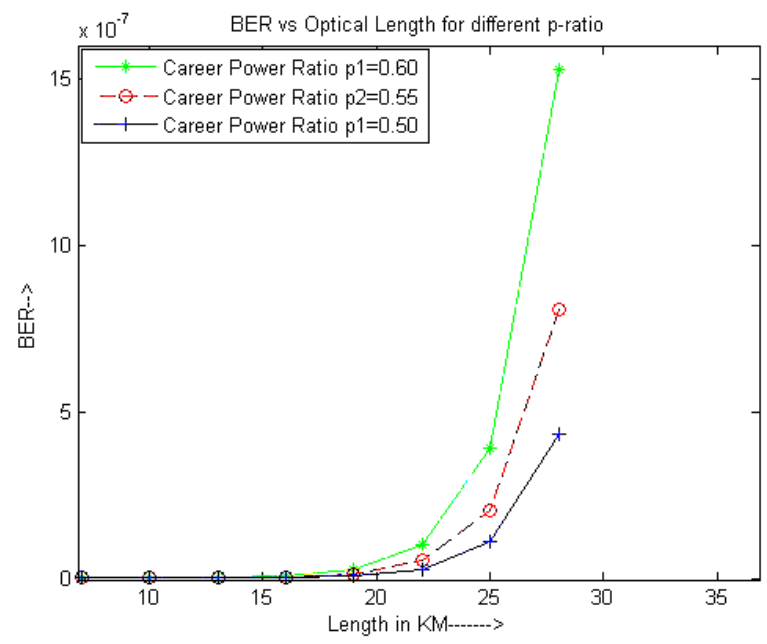

(b)

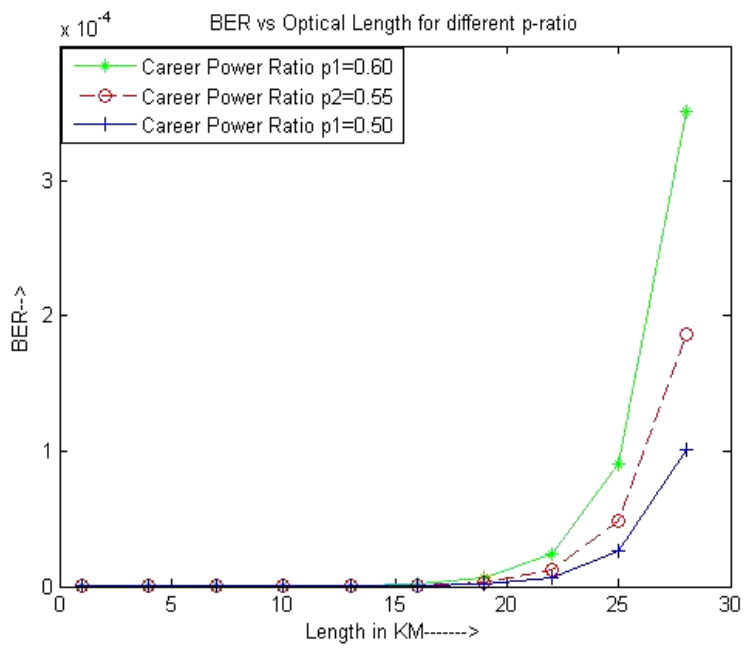

(d)

Figure 6. BER vs optical length for $(a, b) R=1$ and $(c, d) ~ R=0.5$ with $\gamma_{R F}=\pi$. 


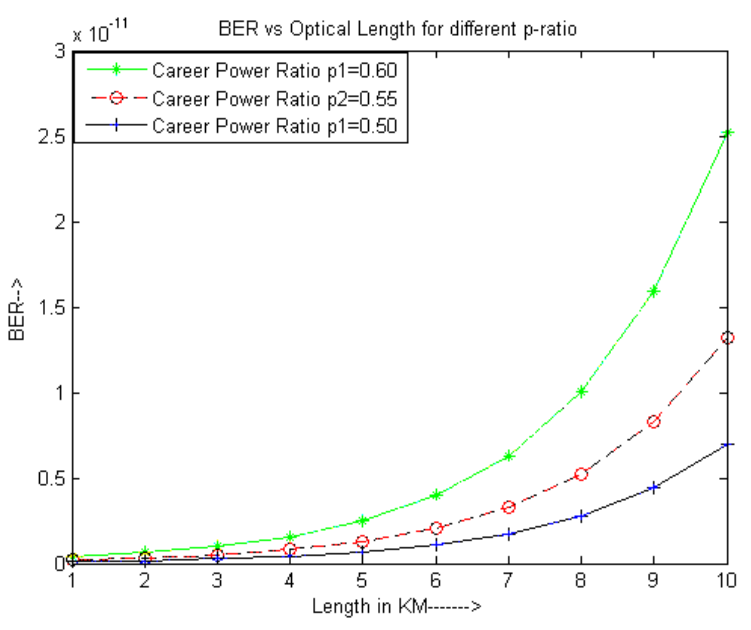

(a)

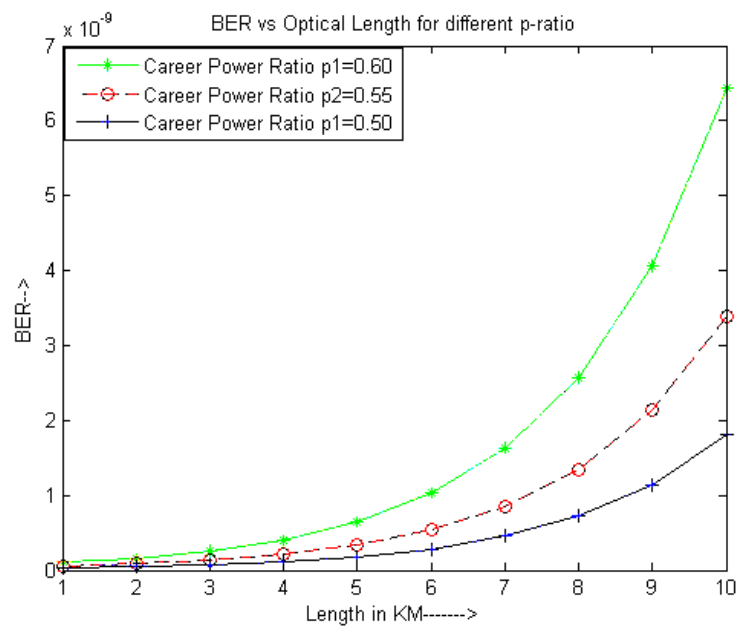

(c)

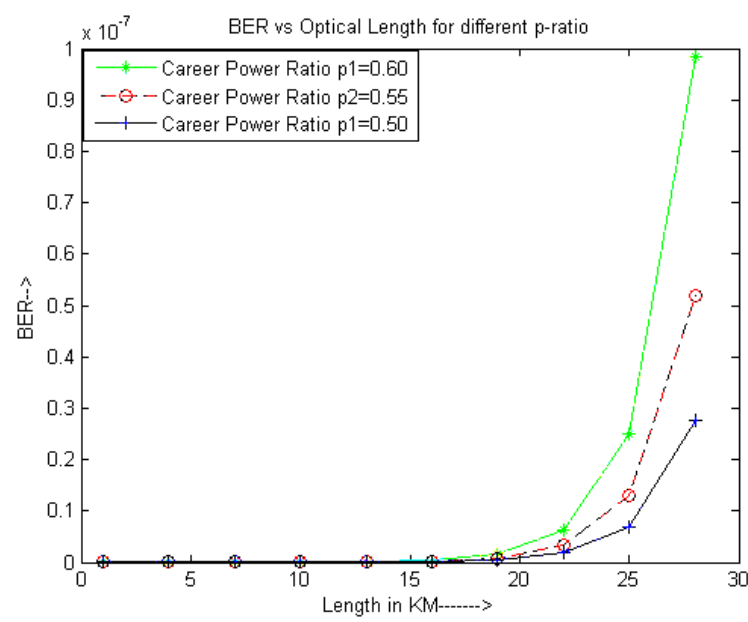

(b)

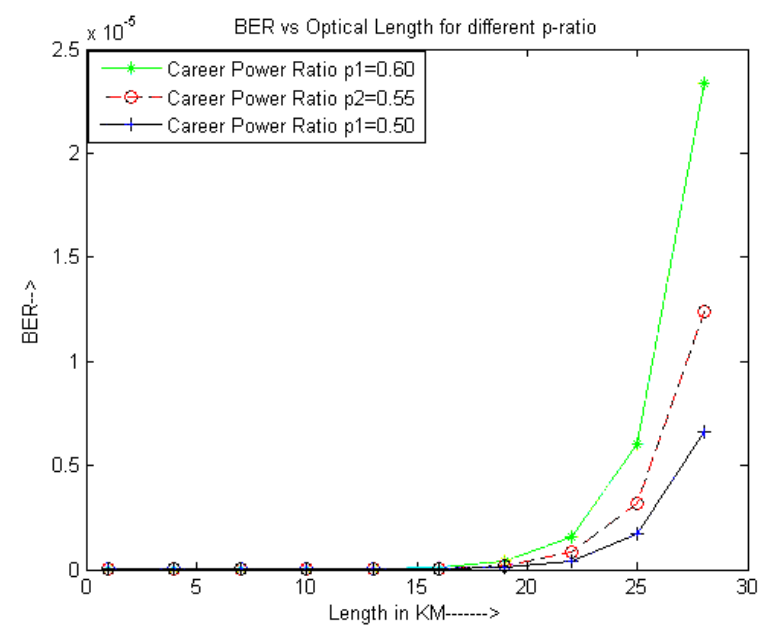

(d)

Figure 7. BER vs optical length for $(\mathbf{a}, \mathbf{b}) \mathbf{R}=1$ and $(\mathrm{c}, \mathbf{d}) \mathbf{R}=\mathbf{0 . 5}$ with $\gamma_{R F}=\pi / 2$.

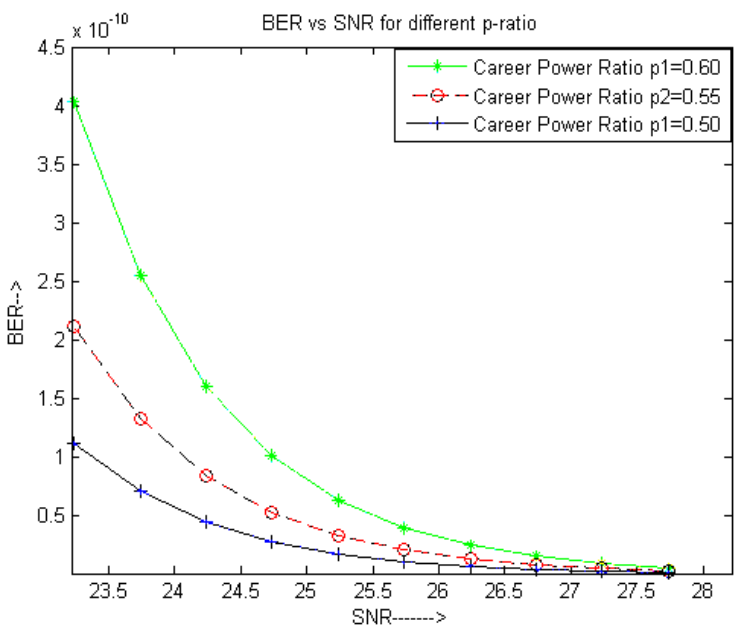

(a)

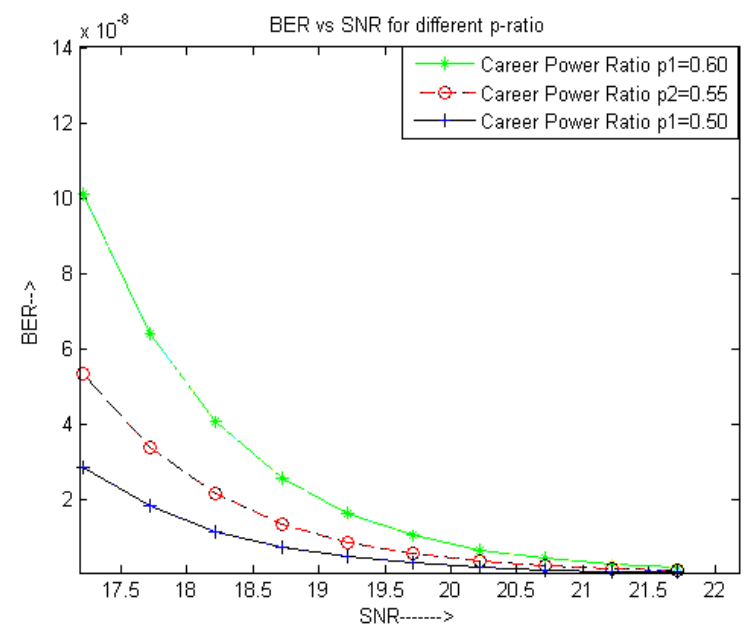

(b)

Figure 8. BER vs OSNR for (a) $R=1$ and (b) $R=0.5$ with $\gamma_{R F}=\pi$. 


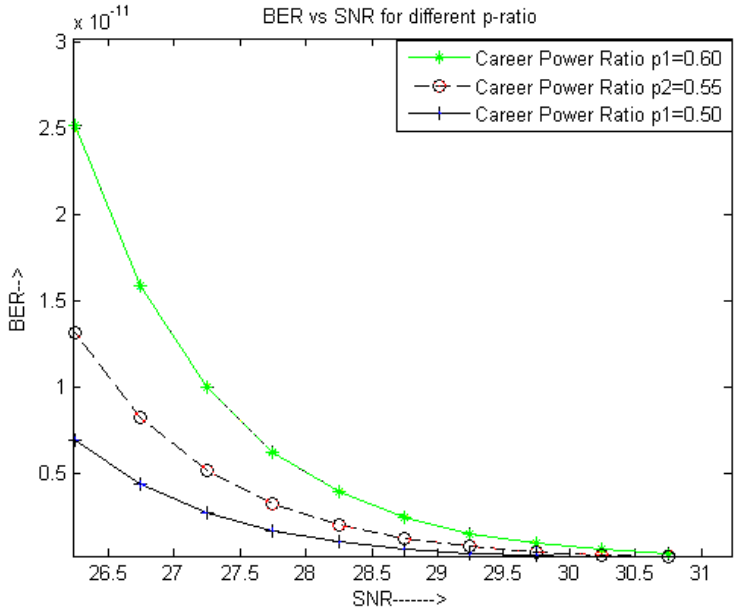

(a)

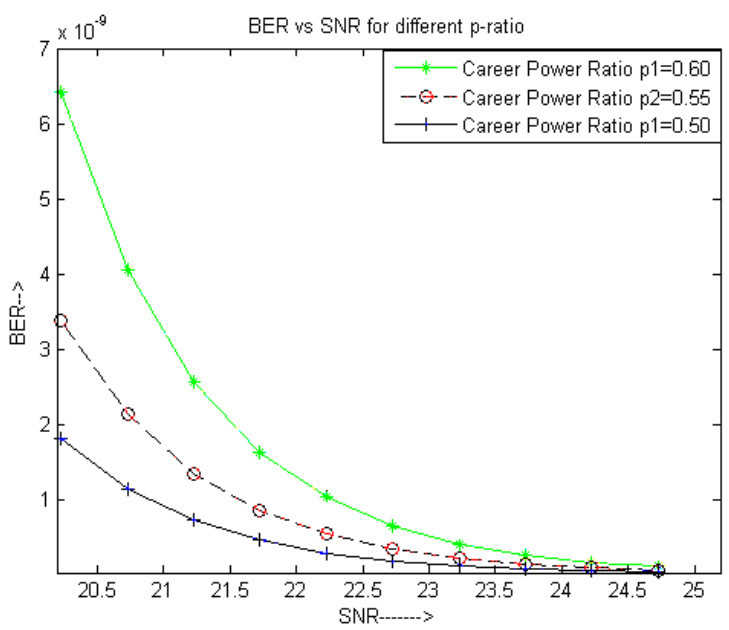

(b)

Figure 9. BER vs OSNR for (a) $R=1$ and (b) $R=0.5$ with $\gamma_{R F}=\pi / 2$.

\section{Conclusions}

In this paper, we have analyzed the impact of p-ratio on OSNR, Q-parameter and BER of RoF communication system at FWHM of RF oscillator linewidth in conjunction with variable value of fiber length $\left(\mathrm{L}_{\text {Fiber }}\right)$ up to 100 $\mathrm{Km}$. An improvement of $3 \mathrm{dBm}$ in OSNR have been observed for p-ratio $=0.5$ and $\gamma_{R F}=\pi / 2$ along with R-parameter of 1. A considerable improvement in Qparameter and BER has also been observed for lesser value of p-ratio.

\section{References}

[1] N. Uesugi, T. Horiguchi, M. Nakazawa and Y. Murakami, "Optical Fiber Cable Measurements in the Field," IEEE Journal on Selected Areas in Communications, Vol. 4, No. 5, 1986, pp.732-736. doi:10.1109/JSAC.1986.1146373

[2] M. Sauer, A. Kobyakov, J. E. Hurley and J. George, "Experimental Study of Radio Frequency Transmission over Standard and High-Bandwidth Multimode Optical Fibers," International Topical Meeting on Microwave Photonics, Seoul, 12-14 October 2005, pp. 99-102. doi:10.1109/MWP.2005.203549

[3] R. J. Green, "Secure Communications: The Infrared Alternative," In ICTON Mediterranean Winter Conference, 6-8 December 2007, pp. 1- 4. doi:10.1109/ICTONMW.2007.4446906
[4] J. Hecht, "Understanding Fiber Optics," $4^{\text {th }}$ Edition, Prentice Hall, Upper Saddle River, 2002.

[5] G. H. Smith and D. Novak, "Overcoming ChromaticDispersion Effects in Fiber-Wireless Systems Incorporating External Modulators," IEEE Transactions on Microwave Theory Technology, Vol. 45, No. 8, 1997, pp. 1410-1415. doi:10.1109/22.618444

[6] T.-S. Cho, C. Yu and K. Kim, "Analysis of CNR Penalty of Radio over Fiber Systems Including the Effects of Phase Noise from Laser and RF Oscillator," Journal of Lightwave Technology, Vol. 23, No. 12, 2005, pp. 40934100 .

[7] J. R. Barry and E. A. Lee, "Performance of Coherent Optical Receivers," Proceeding of Institute of Electrical Engineering, Vol. 78, No. 8, 1990, pp. 1369-1394.

[8] J. Salz, "Modulation and Detection for Coherent Lightwave Communications," IEEE Communication Magagazine, Vol. 24, No. 6, 1986, pp. 38-49. doi:10.1109/MCOM.1986.1093099

[9] V. Sharma, A. Singh and A. K. Sharma, "Analysis the Impact of Laser- and RF Oscillator-Line width over Carrier-to- Noise Ratio in Optical Single Sideband (OSSB) RoF Transmission Systems", Optics and Lasers in Engineering, Vol. 47, No. 11, 2009, pp. 1145-1149. doi:10.1016/j.optlaseng.2009.06.012

[10] U. Gliese, S. Nørskov and T. N. Nielsen, "Chromatic Dispersion in Fiber-Optic Microwave and Millimeter-Wave Links," IEEE Transaction of Microwave Theory Technology, Vol. 44, No. 10, 1996, pp. 1716-1724. doi: $10.1109 / 22.538964$ 